\title{
Doxycycline use in maritime-medical practices
}

\section{Eilif Dahl}

Department of Occupational Medicine, Haukeland University Hospital, the Norwegian Centre for Maritime Medicine, Bergen, Norway

Doxycycline is an antibacterial drug synthetically derived from oxytetracycline. It was approved by the American Food and Drug Administration (FDA) in 1967 [1] and is still used to treat a wide variety of bacterial infections and for the prophylaxis of malaria in short-term travellers ( $<4$ months). To reduce the development of drug-resistant bacteria and maintain effectiveness, FDA recommends that doxycycline should be used only to treat or prevent infections that are proven or strongly suspected to be caused by susceptible bacteria [2]. In the absence of cultures, which is mostly the case in maritime-medical situations, local epidemiology and susceptibility patterns may contribute to the empiric selection of therapy.

Because doxycycline capsules are rather quickly and virtually completely absorbed after oral administration [2], they are popular in the maritime community where parenteral medication is often not readily available. Since bacteriostatic drugs may interfere with the bactericidal action of penicillin, it is advisable to avoid giving doxycycline in conjunction with penicillin [2].

The usual dosage and frequency of administration of doxycycline differs from that of the other tetracyclines. Dose and dosage form may influence the occurrence of adverse effects: Exceeding the recommended dosage may result in an increased incidence of side effects [2], and capsules containing doxycycline hyclate in a powder formulation cause more gastrointestinal side effects like nausea, vomiting, and stomach or abdominal discomfort than capsules containing doxycycline hyclate as coated pellets [3].

Sequential labelling with fluorochromes is a standard technique for the investigation of bone remodelling in vivo. Tetracyclines are the only fluorochromes approved for human application, and - for oral administration - doxycycline is considered for its good bioavailability [4]. Because of their high affinity for calcium, tetracycline is incorporated at the site of active mineralisation of hydroxyapatite. A single oral dose of tetracycline can be traced by fluorescent microscopy in bone biopsies years after application $[5,6]$. With an increasing percentage of female seafarers and a growing population of very young passengers on cruise ships, it is worth recalling that as with all tetracyclines, doxycycline is contraindicated in pregnancy through infancy and childhood up to 8 years of age, except for treatment of anthrax, due to the potential for disrupting bone and tooth development [7]. Even short courses may cause permanent discoloration of the teeth (yellow-grey-brown) and enamel hypoplasia [2].

Although sun block awareness protection has improved in general, patients should be told, particularly in the maritime working environment and in cruise settings, to avoid excessive sunlight while receiving doxycycline because photosensitivity manifested by an exaggerated sunburn reaction is not uncommon. Therapy should be discontinued if phototoxicity (e.g., skin eruption, etc.) occurs [2].

And in such surroundings, where gastrointestinal conditions are common, it is important to keep in mind that absorption of tetracyclines is impaired by antacids and laxatives containing aluminium, calcium, or magnesium, by bismuth subsalicylate, and by iron-containing preparations. Gastrointestinal absorption of doxycycline is reduced up to $20 \%$ by food and/or milk, but this effect is not considered clinically important [2].

In this issue of "International Maritime Health", Rottenstreich, Rottenstreich and Shapira [8] present a naval crewmember who was diagnosed with oesophageal ulcers after taking doxycycline by mouth, thought to be caused by low $\mathrm{pH}$ and slow passage of the capsule through oesophagus. In most previously reported cases horizontal patient position and too little concomitant fluid intake may have been contributing factors, whereas the present patient had been instructed to take the capsule with a glass of water and to sit up for at least 30 min afterward. Compliance regarding unwitnessed self-administration of medication is notoriously uncertain, yet the case is a good reminder that when prescribing doxycycline the patient should be carefully instructed on how to take the capsules. 
The authors also speculate that intake of $330 \mathrm{~mL}$ beer several hours before taking the doxycycline capsule may have contributed to the oesophageal condition [8].

It is safe and - in a maritime setting for a variety of reasons - hardly wrong to advise against mixing alcohol and drugs. But there is no official FDA recommendation to stay away from alcohol during doxycycline therapy. Neither is it stated that the patient should remain in an upright position for a specified time after tablet intake, but it makes sense because in most cases of esophagitis and ulcers tetracycline had been taken in capsule and tablet form immediately before going to bed [2].

Administration of adequate amounts of fluid ("drink fluids liberally") along with capsule and tablet forms of drugs in the tetracycline class is recommended to wash down the drugs and reduce the risk of oesophageal irritation and ulceration. If gastric irritation occurs, it is recommended that doxycycline be given with food or milk, as this does not markedly influence the absorption [2].

Bed-ridden patients tend to sit up and take relatively high doses of doxycycline with a minimal sip of water before immediately reclining. And travellers to primitive tropic locations often receive their malaria prophylaxis weeks or months before starting and have to continue medication for 4 weeks after exposure. Prescription instructions are usually long forgotten and they often consider it easiest - and safest - to take their daily pill at bedtime with a little sip of a disinfecting (alcoholic) beverage just before slipping under the net. Ideally, detailed instructions on how to take the capsules should therefore be particularly stressed person-to-person at prescription and be printed prominently, bold-faced and underlined on the medicine label.

\section{REFERENCES}

(web sites accessed 10-13 August 2015)

1. Doxycycline. https://en.wikipedia.org/wiki/Doxycycline.

2. Doxycycline capsules USP. http://www.accessdata.fda.gov/drugsatfda_docs/label/2012/065055s012lbl.pdf.

3. Bruno A. Citizen petition 2004. www.fda.gov/ohrms/dockets/dailys/04/mar04/031904/04p-0417-cp00001-vol1.pdf.

4. Pautke $\mathrm{C}$, Vogt $\mathrm{S}$, Kreutzer $\mathrm{K}$ et al. Characterization of eight different tetracyclines: advances in fluorescence bone labeling. J Anat 2010; 217: 76-82.

5. Dahl E, Nordal KP, Aksnes L, Halse J. Bone remodeling in predialysis chronic renal failure: How does the choice of index for mineralizing surface influence the interpretation? J Bone Min Res 1989; 4: 845-852.

6. Dahl E, Nordal KP. Renal osteodystrophy in Norway. Aspects of pathogenesis and treatment. (Thesis). University of Oslo, Oslo 1992.

7. Mylonas I. Antibiotic chemotherapy during pregnancy and lactation period: aspects for consideration. Arch Gynecol Obstet 2011; 283 : $7-18$.

8. Rottenstreich M, Rottenstreich M, Shapira S. Doxycycline induced oesophageal ulcers in a navy ship crewmember. Int Marit Health 2015; 66: 181-183. 